
available under aCC-BY-NC-ND 4.0 International license.

\title{
Synthetic amyloid beta does not induce a robust transcriptional response in innate immune cell culture systems
}

\author{
I.Y. Quiroga*1, A.E. Cruikshank ${ }^{* 2}$, K. S. M. Reed ${ }^{3}$, M.L. Bond 3 , B.A. Evangelista ${ }^{4}$, J.H. Tseng ${ }^{4}$, J. V. Ragusa ${ }^{4}$, \\ R. B. Meeker ${ }^{5}$, H. Won ${ }^{6,7}$, S. Cohen ${ }^{4}$, T.J. Cohen ${ }^{5}$, D.H. Phanstiel'1,3,4,\#
}

Alzheimer's disease (AD) is a progressive neurodegenerative disease that impacts nearly 400 million people worldwide. The accumulation of amyloid beta $(\mathrm{A} \beta)$ in the brain has historically been associated with $\mathrm{AD}$, and recent evidence suggests that neuroinflammation plays a central role in its origin and progression. These observations have given rise to the theory that $\mathrm{A} \beta$ is the primary trigger of $\mathrm{AD}$, and induces proinflammatory activation of immune brain cells (i.e. microglia), which culminates in neuronal damage and cognitive decline. In order to test this hypothesis, many in vitro systems have been established to study $\mathrm{A} \beta$-mediated activation of innate immune cells. Nevertheless, the transcriptional resemblance of these models to the microglia in the $\mathrm{AD}$ brain has never been comprehensively studied on a genome-wide scale. To address this, we used bulk RNA-seq to assess the transcriptional differences between in vitro cell types used to model neuroinflammation in $\mathrm{AD}$, including several established, primary and iPSC-derived immune cell lines (macrophages, microglia and astrocytes) and their similarities to primary cells in the AD brain. We then analyzed the transcriptional response of these innate immune cells to synthetic A $\beta$. We found that human induced pluripotent stem cell (hIPSC)-derived microglia (IMGL) are the in vitro cell model that best resembles primary microglia. Surprisingly, synthetic $A \beta$ does not trigger a robust transcriptional response in any of the cellular models analyzed, despite testing a wide variety of $A \beta$ formulations, concentrations, and treatment conditions. Finally, we found that bacterial LPS and INF $\gamma$ activate microglia and induce transcriptional changes similar to those observed in disease associated microglia present in the AD brain, suggesting the potential suitability of this model to study AD-related neuroinflammation.

A lzheimer's disease (AD) affects about 5.8 million people in the USA and nearly 400 million people worldwide. The estimated cost for health care in the United States is 301 billion dollars and this number is expected to increase to 1.1 trillion dollars by 2050 when $\mathrm{AD}$ is projected to affect 13.8 million people ${ }^{1}$. In order to improve diagnosis and treatment options for AD it is imperative to have a better understanding of the molecular mechanisms underlying AD.

The accumulation of Amyloid beta $(A \beta)$ in the brain has been associated with AD since the discovery of this pathology in 1906. This correlation has been supported by a number of findings, including the identification of genetic mutations causing misregulation of $\mathrm{A} \beta$ production and processing as the cause for familiar $\mathrm{AD}^{2}$ and the evidence that identifies $A \beta$ deposition upstream of Tau tangle formation and neuronal death ${ }^{3-8}$. As a result, $A \beta$ deposition has been proposed as the main cause for the development of $\mathrm{AD}$, a theory known as the amyloid cascade hypothesis. Nevertheless, recent findings have raised questions about this hypothesis, including the failure of drug trials targeting $A \beta$ accumulation and the observation of similar patterns of $A \beta$ deposition in $A D$ and healthy brains ${ }^{9}$. This evidence suggests that although closely associated, $A \beta$ accumulation might not be the only causal factor for the development of $\mathrm{AD}$ and other elements might contribute to a more complex etiology.

Evidence pointing to chronic neuroinflammation as a

\footnotetext{
*Equal contribution

'University of North Carolina, Thurston Arthritis Research Center, Chapel Hill, NC, United States of America

${ }^{2}$ University of North Carolina, Postbaccalaureate Research Education Program, Chapel Hill, NC, United States of America

${ }^{3}$ University of North Carolina, Curriculum in Genetics \& Molecular Biology, Chapel Hill, NC, United States of America

${ }^{4}$ University of North Carolina, Department of Cell Biology and Physiology, Chapel Hill, NC, United States of America

${ }^{5}$ University of North Carolina, Department of Neurology, Chapel Hill, NC, United States of America

${ }^{6}$ University of North Carolina, Department of Genetics and Neuroscience Center, Chapel Hill, NC, United States of America.

${ }^{7}$ University of North Carolina, Neuroscience Center, Chapel Hill, NC, United States of America

\#Contact: douglas_phanstiel@med.unc.edu
} 

available under aCC-BY-NC-ND 4.0 International license.
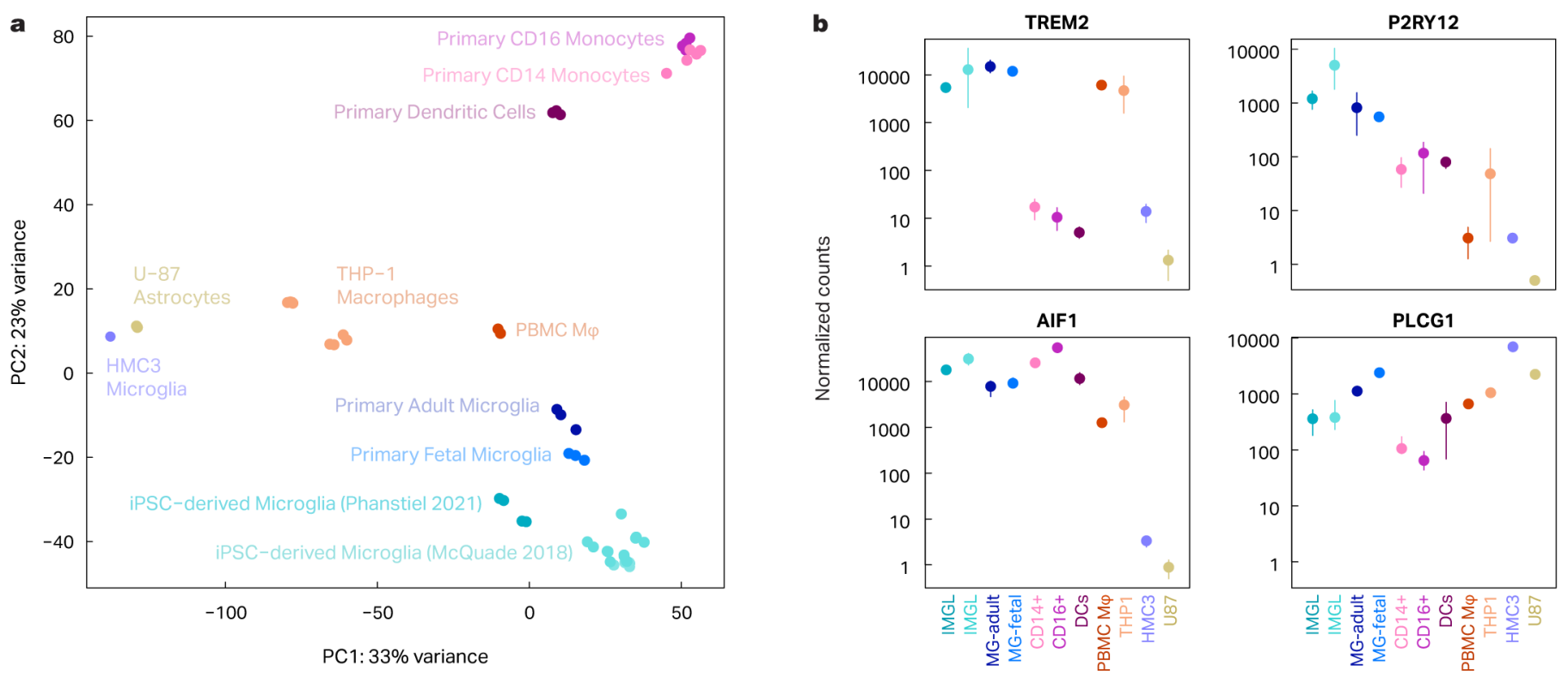

Fig. 1 | IMGL are the in vitro cell cultures that best resemble human microglia. Bulk RNA-seq was performed in different innate immune in vitro cell cultures and compared to already published data from related cell types. (a) Principal component analysis demonstrates that IMGL (light and dark turquoise) is the cell type that clusters most closely to cultured human fetal and adult microglia (dark and light blue). Additionally, these cells are distinct from human CD14+ monocytes (light pink) and CD16+ inflammatory monocytes (medium purple), and dendritic cells (dark purple). PBMC M $\varphi$ (red) and THP-1 cells (orange) are next in similarity and surprisingly the microglia HMC3 cell line (light purple) clusters more closely to the astrocytoma U-87 cell line (mustard) than to any microglia cell type analyzed. (b) The analysis of normalized counts of a subset of microglia markers show similar trends.

crucial contributor to AD progression has accumulated over the last decade. Patients with AD have higher levels of neuroinflammation when analysing inflammatory markers in postmortem AD brains or live neuroimaging $^{10-15}$. Resident brain immune cells from patients with AD express more genes associated with immune pathways and phagocytosis than controls indicating a state of chronic activation ${ }^{16}$. Finally, recent GWAS studies revealed enrichment of disease-associated variants at monocyte, macrophage, and microglia regulatory loci implicating innate immune cells as key mediators of AD risk ${ }^{17-19}$. This evidence has led to the hypothesis that in the Alzheimer's brain, $A \beta$ triggers an exacerbated proinflammatory activation of brain innate immune cells, particularly microglia and to a lesser extent astrocytes, resulting in a chronic neuroinflammatory state that causes neuronal damage and cognitive decline.

Several in vitro models have been established to further investigate the role of immune cells in $\mathrm{AD}$ and the functional consequences of AD risk genes in these cells. Many of these models involve the exposure of mice or human innate immune cell types-either primary cells, established cell lines or human induced pluripotent stem cell (hIPSC)-derived microglia (IMGLs) to synthetic A $\beta$. It has been assumed that these in vitro scenarios would trigger an inflammatory response similar to the one observed in the human $\mathrm{AD}$ brain, but to the best of our knowledge comprehensive profiles of the transcriptional response to this stimulus in vitro have not been reported. Here we assess the transcriptional differences between in vitro cell types broadly used to model neuroinflammationincluding THP-1 macrophages, human peripheral blood mononuclear cell derived macrophages (PBMC M $\phi$ ), U-87 Astrocytes, HMC3 microglia-like cells, and IMGLs-and study their transcriptional response to synthetic $A \beta$. We found that IMGLs are the in vitro cell model that best resembles primary microglia, but that synthetic $A \beta$ does not trigger a robust transcriptional response in any of the cells analyzed. Finally we found that treatment of IMGLS with bacterial LPS/INF $\gamma$ induces transcriptional changes similar to those observed in disease associated microglia present in the $\mathrm{AD}$ brain, suggesting the potential suitability of this model to study AD-related neuroinflammation.

\section{Results}

\section{Transcriptional comparison of cultured cell types to study AD}

In order to determine which of the currently available and most widely used in vitro immune models best resembles the transcriptional signature of human microglia, we performed RNA-seq in PBMC M $\phi$, THP-1 macrophages, HMC3 microglia, U-87 astrocytoma cells, and IMGLs. We used principal component analysis (PCA) to compare the transcriptomic profiles of these cells with already published data from primary human adult and fetal microglia as well as with other related immune cell types $^{20}$. Our results showed that IMGLs cluster closely to 
bioRxiv preprint doi: https://doi.org/10.1101/2021.09.14.460110; this version posted September 15, 2021. The copyright holder for this preprint (which was not certified by peer review) is the author/funder, who has granted bioRxiv a license to display the preprint in perpetuity. It is made available under aCC-BY-NC-ND 4.0 International license.
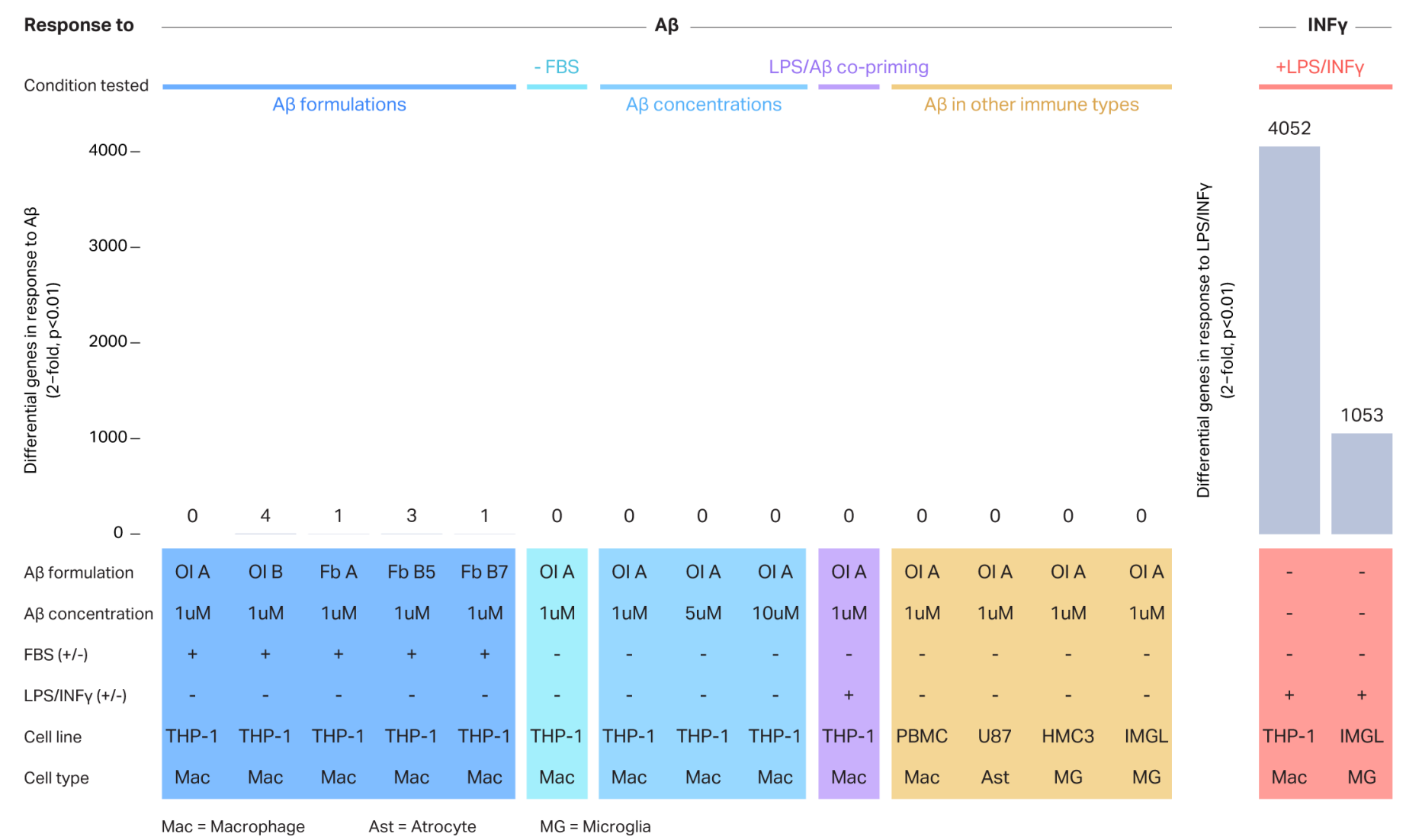

Fig. 2 | Transcriptional response of immune in vitro cell cultures exposed to different proinflammatory stimuli. Bulk RNA-seq was performed in different innate immune in vitro cell cultures exposed to proinflammatory stimuli during $24 \mathrm{~h}$. THP-1 cells were treated with different $A \beta$ formulations including $A \beta$ oligomers $(\mathrm{OI})$ and $A \beta$ fibrils ( $\mathrm{Fb}$ ) obtained using different protocols (dark blue); $A \beta$ in the absence of FBS (turquoise), with different $A \beta$ concentrations (light blue), or co-priming using LPS/ INFy and A $\beta$ together (purple). Additionally, other immune cell types were treated with $A \beta$ (orange). Lastly THP-1 cells and IMGLs were treated with $10 \mathrm{nM}$ LPS+20nM INFy (red). Differential genes in response to A 3 or LPS/ INFy were determined by comparing to condition-matched controls using DESeq2, $p<0.01$, absolute fold change $>2$.

both human adult and fetal microglia and further away from other primary immune cells such as monocytes and dendritic cells (Figure 1A). PBMC M $\phi$ and THP-1 macrophages are the next closest in proximity while the HMC3 microglia established cell line has the least similarities to primary microglia and, surprisingly, clusters almost perfectly with the U-87 astrocytoma cell line. We used variance partitioning to determine how much of this clustering could be driven by the fact that the data from these cell types was generated by two separate labs (variancePartition ${ }^{21}$ ). We calculated the percentage of variance explained by cell type, lab of origin, biological replicate, and technical replicate and found that on average $71 \%$ of variance was due to differences in cell type, while only $12 \%$ was attributable to lab of origin. Investigation of microglia marker genes in each of these cell types further confirmed the similarity between IMGLs and primary microglia (Figure 1B). IMGLs showed high expression of TREM2, P2RY12, AIF1 (IBA1) and PLCG1, similar to primary adult and fetal microglia. THP-1 macrophages exhibited the next most similar pattern of expression at most but not all genes, while the HMC3 microglia established cell line exhibited low expression for the majority of these microglia marker genes. These results suggest that, among the cells investigated, IMGLs best resemble primary human microglia and are a suitable in vitro model to study neuroinflammation.

\section{Synthetic $A \beta$ does not induce a robust transcriptional response}

We next sought to characterize the global transcriptional response of IMGLs to synthetic $A \beta$ to model the changes that occur in microglia in the AD brain. We treated IMGLs with $1 \mu \mathrm{M}$ oligomeric $A \beta$ for 24 hours and performed stranded, paired-end, total RNA-seq. Surprisingly, we observed no significant changes in gene expression in response to $A \beta$ treatment (DESeq2, $p<0.01$, absolute fold change $>2$ ) (Figure 2). To explore the degree to which treatment conditions affect these results, we turned to THP-1 cells which are a more tractable system and express many microglia marker genes such as TREM2, a cell surface receptor known to bind $A \beta^{22}$.

We prepared synthetic $A \beta$ in the form of oligomers or fibrils following two different previously published protocols: protocol A described by Tseng et $\mathrm{al}^{23}$ or protocol B described by Abud et al ${ }^{24}$. Successful generation of oligo- 

available under aCC-BY-NC-ND 4.0 International license.

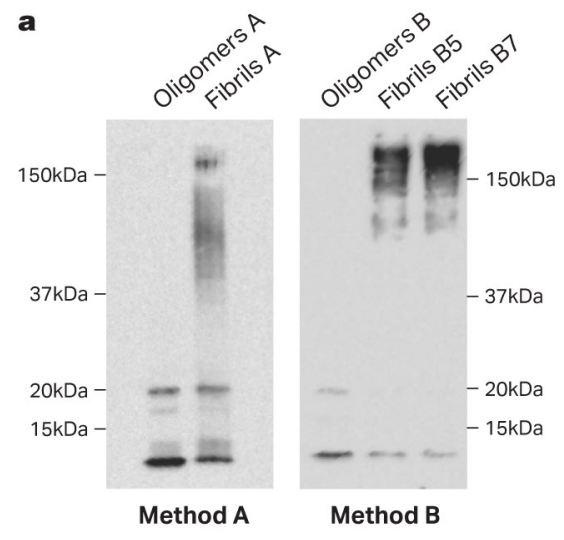

b

THP-1 Macrophages
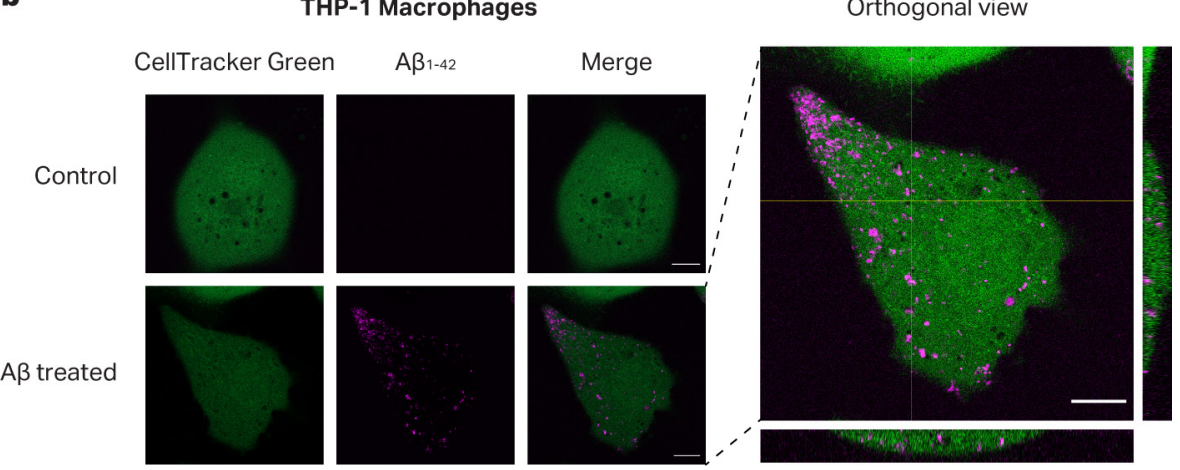

Fig. 3 | A $\beta$ is incorporated into THP-1 cells. (a) A $\beta$ Oligomers and fibrils were generated using either Tseng et al, 2017 (Method a) or Abud et al. 2017 (Method b) protocol. In the last case, fibrils were generated during either 5 or 7 days of incubation (B5 and B7, respectively). 120 ng of $A \beta$ were run in an electrophoresis on a $12 \%$ SDS-PAGE followed by western blot using anti-A $\beta$ primary antibody (6E10 Biolegend). (b) THP-1 cells were labeled

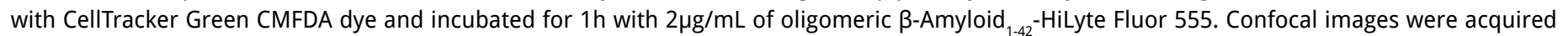
on an inverted Zeiss 800 laser scanning confocal microscope. Scale bar, $10 \mu \mathrm{m}$.

mers and fibrils was confirmed by western-blot (Figure 3A). We also confirmed that both THP-1 cells and IMGLs are capable of phagocytosing synthetic $A \beta$ after $1 \mathrm{~h}$ of incubation (Figure 3B, Supplementary Figure 1).

Next, we proceeded to treat THP-1 cells with $A \beta$ under various conditions and performed RNA-seq to analyze the global transcriptional response. As observed previously in IMGLs, when THP-1 macrophages were treated with the different $A \beta$ formulations (Figure 2, dark blue), no robust transcriptional response was observed in any condition. From this point forward, we utilized the oligomeric form of $A \beta$ since it has been previously described as the more "active" form of $A \beta^{25-27}$. We next eliminated the presence of fetal bovine serum (FBS) during the treatment to determine whether it was interfering with the $A \beta$ incorporation, since it has been suggested that the albumin present in the FBS could act by "sequestering" the soluble $A \beta$ in the media, reducing the availability of $A \beta$ capable of binding the cellular receptors ${ }^{28}$. No significant transcriptional response was seen in the absence of FBS during the A $\beta$ treatment. We then decided to test different concentrations of $A \beta$. Again, no response was seen with any of the $\mathrm{A} \beta$ concentrations tested ranging from 1 to $10 \mu \mathrm{M}$. Following the relatively new hypothesis that microbial presence in the brain could be a contributing factor to the development of $\mathrm{AD}$ in addition to $\mathrm{A} \beta$ accumulation ${ }^{29-32}$, we decided to test whether $A \beta$ has any synergistic effect when the cells were co-primed with LPS and INF $\gamma$. We found no significant differences when treating THP-1 cells with this combination compared to LPS/INF $\gamma$ alone. Additionally, we tested whether $A \beta$ had a significant effect in any of the other innate immune cell cultures used previously. No significant transcriptional response to $A \beta$ was seen in either PBMC M $\phi$, U-87, or HMC3 cells. As an alternative method to activate the inflammasome and also as a positive control for proinflammatory activation, we treated
THP-1 cells with LPS and INF $\gamma$. As expected, THP-1 cells treated with this stimulus displayed a robust transcriptional response, (DESeq2, 4053 differential genes, $p<0.01$, absolute fold change $>2$ ) (Figure 3) Finally, we treated IMGL with LPS and INF $\gamma$ and observed a robust transcriptional response (DESeq2, 1053 differential genes, $p<0.01$, absolute fold change $>2$ ) although smaller to the one observed in THP-1 macrophages.

\section{LPS/INF $\gamma$ activation of IMGLs mirrors transcriptional changes seen in DAM}

In contrast to amyloid beta, and in agreement with a recent study ${ }^{33}$, LPS/INF $\gamma$ treatment induced a robust transcriptional response in both THP-1 macrophages and IMGLs (Figure 4A-B). Upregulated genes included key proinflammatory genes IL1B, CD38, and CCL2 and were enriched for classical inflammation pathways including NF- $\kappa B$ and Toll-Like Receptor Signaling (Homer, $p<0.05$ ). Because many of these genes and pathways are upregulated in $\mathrm{AD}$ microglia and because $\mathrm{AD}$ pathogenesis has been associated with viral and bacterial infection ${ }^{32,34,35}$, some groups have used LPS or LPS/INF $\gamma$ to stimulate innate immune cells either in vitro or by direct injection in animal's brains as a model of AD-associated neuroinflammation ${ }^{36,37}$.

To assess the validity of LPS/INF $\gamma$ treated IMGLs as a model of $\mathrm{AD}$, we compared the transcriptional profiles of our LPS/INF $\gamma$-treated IMGLs to published DAM gene signatures from $\mathrm{AD}$ mouse models ${ }^{38}$. DAM genes were significantly enriched among LPS/INF $\gamma$ differentially expressed genes (Fisher's Exact Test, $\mathrm{p}=4.17 \times 10^{-15}$ ). The directional effects of our LPS/INF $\gamma$-treated IMGLs were also concordant with the directional effects of the DAM signature (Figure 5). Genes upregulated in DAM tended to be upregulated in IMGLs upon treatment with LPS/INF $\gamma$ (median log2 fold-change $=0.26$, Wilcoxon test, $p=1.46 \times 10^{-6}$ ), 
a

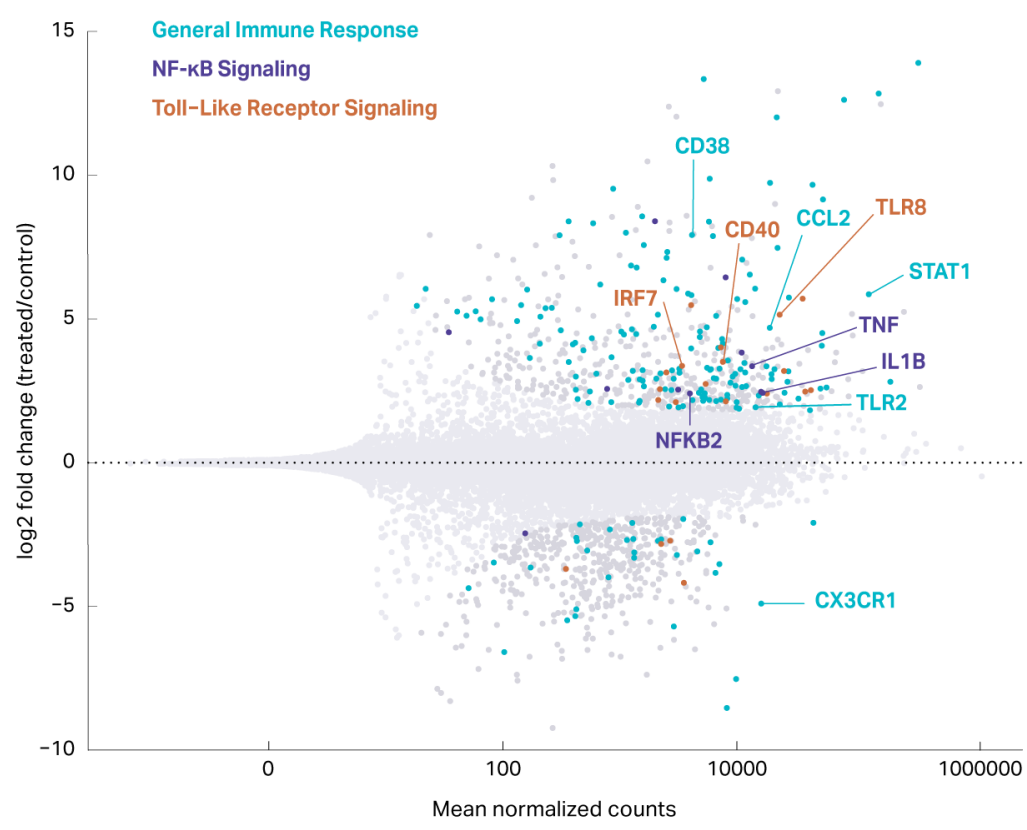

b

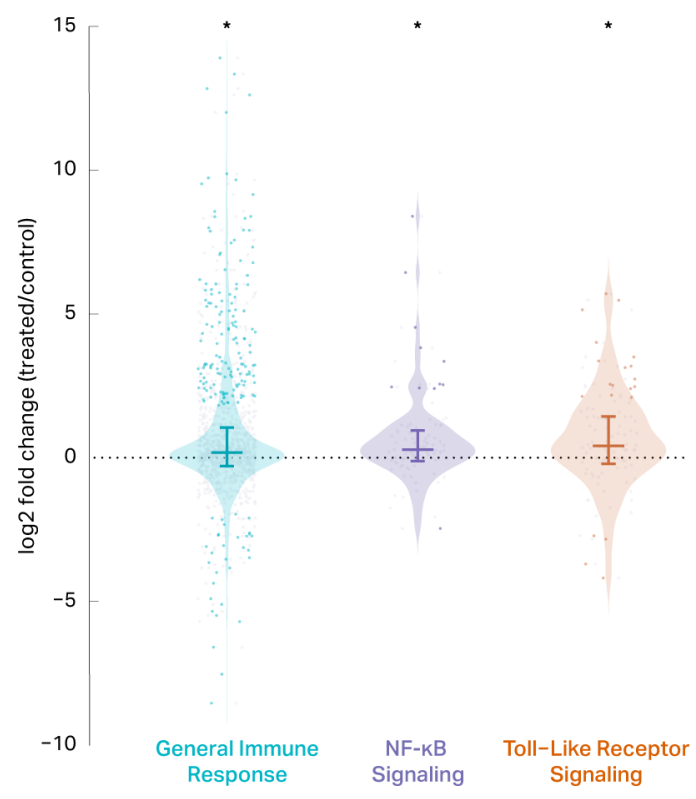

Fig. 4 | LPS/INFy treatment induces a robust proinflammatory transcriptional change in IMGLs. (a) Bulk RNASeq was performed in IMGLS treated with $1 \mu \mathrm{M} \mathrm{A} \beta$ oligomers or $10 \mathrm{nM}$ LPS $+20 \mathrm{nM}$ INFy for $24 \mathrm{~h}$. Differential genes $(p<0.01$, absolute fold change $>2)$ are labeled in dark gray and non-significant genes $(p>0.01$, absolute fold change $<2$ ) are labeled in light gray. Blue, purple, and orange represent differential genes associated with general immune response, NF-kB signaling, and toll-like receptor signaling respectively. (b) $\log _{2}$ fold-changes for the genes in specific pathways shown in 4A. Non-significant genes are shown in light gray and differential genes are shown in blue, purple, and orange respectively for general immune response, NF-kB signaling, and toll-like receptor signaling. 1 Sample Wilcoxon test showed significant differences for the genes in each pathway vs $0\left(p=6.38 \times 10^{-18}, p=1.28 \times 10^{-4}, p=1.88 \times 10^{-4}\right.$ respectively $)$.

while genes that are downregulated in DAM also tended to be downregulated in IMGLs upon treatment with LPS/ INF $\gamma$ albeit to a lesser degree (median log2 fold-change $=$ -0.09 , Wilcoxon test, $\mathrm{p}=0.02$ ) (Figure 5). Among the shared upregulated genes are MS4A7, which has been shown to regulate TREM2 protein and to be upregulated in late-onset $\mathrm{AD}$ brains ${ }^{39,40}$, and ITGAX which has been shown to be upregulated in aged microglia ${ }^{41,42}$. We note that the effect sizes of LPS/INFy on DAM genes are small; however, these effect sizes are comparable to those observed in the mouse models used to identify DAM genes (median log2 fold-change of 0.17 and -0.23 for upregulated and downregulated DAM genes respectively). Taken together, these results suggest that LPS/INF $\gamma$-treated IMGLs recapitulate many features of the DAM transcriptome.

\section{Discussion}

Progress to unveil the mechanisms behind the origin and progression of $\mathrm{AD}$ has been slow in part due to the difficulty in finding adequate models to mimic this disease in the laboratory. Although mouse models have shown to be useful in many aspects, the genetic and physiological differences between species have limited certain areas of research ${ }^{43}$. Conversely, access to human brain samples is limited and the molecular identity and stability of some cell types, mostly brain immune cells, once extracted from the brain have been controversial ${ }^{44,45}$. As a result, the development of human in vitro cell culture models, particularly IPSC-derived celular models, have been broadly used to mimic $\mathrm{AD}$ in a dish ${ }^{46-48}$. This alternative has been particularly valuable since it is amenable to genome engineering, disease modeling, and high-throughput screening techniques.

Here, we analyzed the feasibility of using these in vitro cell models, including several established primary and iPSC-derived immune cell lines, and their similarity to human microglia using a genome-wide transcriptomic approach. Not surprisingly, we identified IMGLs as the most similar in vitro culture to mimic human primary microglia (Figure 1). This agrees with previous studies done both using RNA-seq ${ }^{20,24}$ or microarray and qPCR approaches with fewer cell types ${ }^{49,50}$.

When the use of IMGLs is not possible due the complexity and high costs of their obtention, our results also suggested that the use of PBMC M $\phi$ or even macrophages derived from the THP-1 cells are preferable before the use of the HMC3 microglia established cell line, which has fewer similarities with primary microglia. Interestingly, a poor transcriptional similarity between a murine established microglia cell line (BV2) and primary microglia has also been previously reported ${ }^{51}$, raising concerns about 

available under aCC-BY-NC-ND 4.0 International license.

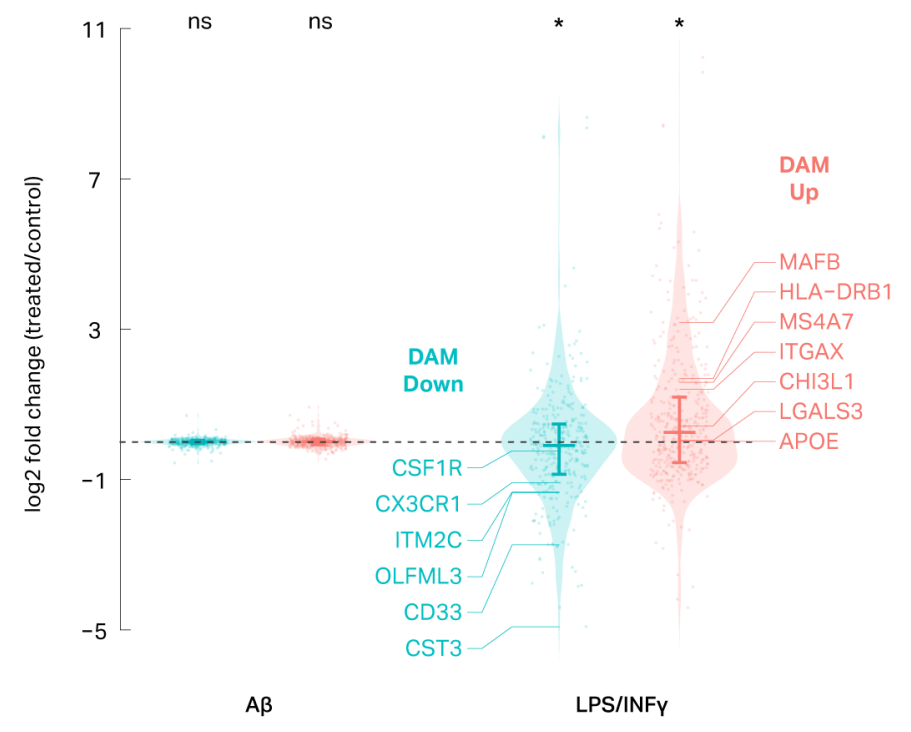

Fig. 5 | Treatment of IMGLs with LPS/INFy induces transcriptional changes similar to those observed in disease associated microglia. Violin plots depict how DAM genes change in IMGLs treated with $A \beta$ (left) or LPS + INFy (right). DAM down and DAM up genes are significantly down and upregulated, respectively, in IMGLs treated with LPS + INFy (Wilcoxon test $p=0.02$ and $p=1.46 \times 10^{-6}$ respectively).

the established microglia cell lines that are most often selected to study neuroinflammation in neurodegenerative diseases.

The stimulation of different innate immune cells in culture with synthetic $A \beta$ has become a common approach to model $\mathrm{AD}$ in vitro, as $\mathrm{A} \beta$ accumulation in the brain is considered one of the main triggers for the development of $\mathrm{AD}$ and immune brain cells have been identified as the main players in AD origin and progression. Although the phagocytosis of this component into in vitro microglia-like cell cultures has been previously shown ${ }^{24,52}$, very few studies have shown any kind of pro-inflammatory response after this treatment. And those studies that did investigate inflammation focused on only a handful of proinflammatory genes and proteins quantified via qPCR, micorarrays, or western blots ${ }^{24,53-55}$.

Here we analyzed the effect of synthetic $A \beta$ treatment in innate immune in vitro cell cultures using the same genome-wide transcriptomic approach with a variety of experimental conditions. To our surprise, even though $A \beta$ is phagocytosed inside the cells (Figure 3B), we found that it does not trigger a robust transcriptional response in any of the tested cell types or conditions (Figure 2).

Several factors could explain why we did not observe robust transcriptional activation in these experiments. First, the synthetic $A \beta$ used here has known differences in structure, heterogeneity and toxicity compared with the $A \beta$ found in human $A D$ brains. $A \beta$ isolated from $A D$ patients comprises a complex mix of $A \beta$ peptides with heterogeneous lengths and posttranslational modifications, ${ }^{27,56-62}$. The use of $A \beta$ preparations extracted from postmortem human brains using previously published extraction protocols ${ }^{63}$ could give more insight into this hypothesis in future studies. Second, the microglia response to $A \beta$ and the consequent neurotoxic effect could be driven uniquely by post transcriptional events, which would agree with previous reports describing the relevance of this layer of regulation in the control of the innate immune response ${ }^{64-66}$ and previous reports of increased proinflammatory cytokine release after $A \beta$ treatment ${ }^{53,67}$. Third, these mono cultured cell lines may not accurately reflect the biology of a human brain which involves a vast array of intertwined cell types. Indeed, studies in animal models have revealed inflammation and microglial activation following injection of synthetic $A \beta^{68-70}$. More work needs to be done to determine if a similar response is observed in brain organoids.

Regardless of these possible explanations, our results suggest that using in vitro immune cell cultures in the presence of synthetic $A \beta$ might not be an adequate and/ or sufficient model to mimic the microglia response in the context of the $\mathrm{AD}$ brain. Moreover, this suggests that $\mathrm{A} \beta$ phagocytosis alone is not sufficient evidence of microglia activation, at least at the transcriptional level.

Even though these results do not disprove the hypothesis that in the human brain, $A \beta$ induces an exacerbated inflammatory response driven by brain immune cells, and that this plays a major role in the origin and progression of $\mathrm{AD}$, it adds evidence that supports the current concern about placing the $A \beta$ cascade hypothesis as the lone explanation for the $\mathrm{AD}$ etiology ${ }^{71}$. More factors, such as the role of tau, microbial infections, and more complex interactions between different brain cell types should be considered and further investigated to understand the origins of the neuroinflammation state observed in the $\mathrm{AD}$ brain.

Finally, in agreement with recent studies ${ }^{33}$, we showed that IMGLs exhibit a significant transcriptional response to LPS/INF $\gamma$. While this response is smaller than the one observed in THP-1 cells, as was also previously reported ${ }^{50}$, it resembles the transcriptional profile of DAMs in the AD brain. These results suggest that treating IMGLs with LPS/ INF $\gamma$ may be a suitable platform to study AD-related microglia inflammation.

\section{Methods}

\section{Amyloid beta formation}

Amyloid-beta $\left(\mathrm{A} \beta_{1-42}\right)$ (AnaSpec; Fremont, CA) was prepared using two different methods as indicated in the figure legends (Methods A or B). When not explicitly indicated, $A \beta$ generated following Method $A$ was used. In the Method A A $\beta$ was generated as previously described ${ }^{23}$. Amyloid-beta $\left(A \beta_{1-42}\right)$ peptide (AnaSpec; Fremont, $C A$ ) was dissolved in Hexafluoro-2-propanol (HFIP, Sigma) to 1mM, 
incubated for 1 hour, then left to evaporate in a vacuum concentrator overnight allowing monomerization. To form oligomerized $A \beta(O A \beta)$, the dried film was reconstituted in DMSO (Sigma) to a concentration of $5 \mathrm{mM}$, and then diluted to $15 \mu \mathrm{M}$ in PBS (pH 7.4) and stored at room temperature for 30 minutes. To form fibrillarized $A \beta$ (fA $\beta$ ), the dried film was reconstituted in a $40 \mathrm{mM}$ Tris- $\mathrm{HCl}(\mathrm{pH} 8.0)$ and $250 \mathrm{mM} \mathrm{NaCl}$ solution, incubated at room temperature with agitation for 6 days, then centrifuged for 10 mins at $15000 \mathrm{~g}$. The resulting pellet was resuspended in PBS to a concentration of $15 \mu \mathrm{M}$. OA $\beta$ and $\mathrm{fA} \beta$ formation were confirmed by western blot as it is described below. $A \beta$ was thoroughly mixed and diluted in media to the desired concentration prior to cell exposure. FA $\beta$ contained some oligomers as indicated by western blot analysis.

In method $B, A \beta$ was reconstituted as previously described in Abud, 2017. Briefly, $A \beta$ was dissolved in 75ul of $\mathrm{NH} 4 \mathrm{OH}(0.1 \%)$ then filled to $1 \mathrm{ml}$ with sterile $\mathrm{PBS}$ to achieve a $1 \mathrm{mg} / \mathrm{ml}$ concentration. $\mathrm{A} \beta$ was then diluted to $100 \mu \mathrm{g} / \mathrm{ml}$ with sterile water, vortexed, and incubated at $37^{\circ} \mathrm{C}$ for 30 mins to form oligomerized $A \beta$ (oA $\beta$ ), and 7 days or 5 days to form fibrillarized $A \beta$ (fA $\beta$ ). $A \beta$ was thoroughly mixed and diluted in cell culture media to the desired concentration prior to cell exposure. FA $\beta$ contained some oligomers as indicated by western blot analysis (Figure 2A).

\section{Characterization of $A \beta$ using Western blot}

The size of the resulting $A \beta$ preparation after performing each protocol was characterized using SDS-PAGE and western blotting. $120 \mathrm{ng}$ of proteins were separated by electrophoresis in a $12 \%$ gel and transferred to a nitrocellulose blotting membrane. After blocking, the membranes were incubated overnight at $4^{\circ} \mathrm{C}$ with anti-A $\beta$ primary antibody (6E10 Biolegend, SIG-39320, 1:1000) following incubation with goat anti-mouse secondary antibody (Thermo Scientific, 1:1000) during $1 \mathrm{~h}$. The membranes were subsequently washed and placed in the Radiance Plus Chemiluminescent Substrate (Azure Biosystems, Dublin, CA, USA) and visualized using the Azure c600 gel imaging system (Azure Biosystems).

\section{Immortalized human cell lines}

The immortalized human monocyte cell line THP-1 (ATCC, \#:ATCC ® TIB-202TM, obtained from the Tissue culture facility at UNC Chapel Hill), was cultured in RPMI 1640 with L-Glutamine-Bicarbonate (Sigma-Aldrich \# R8758) containing 10\% FBS (Thermo Fisher Scientific, GibcoTM \# 10500064), and 1\% penicillin/streptomycin (Sigma-Aldrich \#P4333) in an atmosphere of $5 \% \mathrm{CO}_{2}$ at $37^{\circ} \mathrm{C}$. To differentiate THP-1 monocytes into macrophages, cells were plated at a density of $1.0 \times 10^{6}$ cells/well in 6-well plates and incubated with $25 \mu \mathrm{M}$ PMA (12-O-tetradecanoylphorbol) during $24 \mathrm{~h}$. Macrophages were then allowed to rest for $72 \mathrm{~h}$ before $A \beta$ or LPS/INF $\gamma$ treatment as described below. The HMC3 immortalized microglia cell line (obtained from ATCC \# CRL-3304) and the astrocyte-like U-87 cell line (ATCC \# 30-2003 obtained from the Tissue culture facility at UNC-Chapel Hill) were both cultured in EMEM (ATCC \# 30-2003) containing 10\% FBS and 1\% penicillin/ streptomycin in an atmosphere of $5 \% \mathrm{CO}_{2}$ at $37^{\circ} \mathrm{C}$.

Cells were treated with $1 \mu \mathrm{M} \mathrm{A} \beta$ or $10 \mathrm{nM}$ LPS/20nM INFy (Sigma-Aldrich \# L2630 /Peprotech \# 300-02) during $24 \mathrm{~h}$. Treatments were carried on in serum-free media with $1 \%$ penicillin/streptomycin $(100 \mathrm{U} / \mathrm{ml}$ penicillin/100 $\mu \mathrm{g} / \mathrm{ml}$ streptomycin) unless otherwise stated.

\section{Generation of peripheral blood mononuclear cell- derived macrophages}

Human peripheral blood mononuclear cells (PBMCs) were isolated from deidentified healthy donor blood obtained from the New York Blood Center (Long Island City, NY). Research was approved by the center prior to acquisition. Approximately $10^{8}$ PBMCs were isolated using Ficoll-Paque density gradient centrifugation (MilliPore Sigma \# GE17-5442-02). Cells were cultured in DMEM $10 \%$ FBS and seeded in 6-well ultra-low adhesion plastic culture dishes. Monocytes were allowed to adhere for a period of 5-7 days in vitro. Media was then exchanged to DMEM containing $15 \mathrm{ng} / \mathrm{mL}$ recombinant human granulocyte-macrophage colony stimulating factor (rhGM-CSF; R\&D Systems \# 215-GM-010/CF). PBMC M $\phi$ were allowed to differentiate for an additional 5 days and were returned to DMEM 10\% FBS until stimulation.

\section{Generation of IPSCs-derived microglia}

IPSCs-derived microglia cells were generated as previously described (McQuade et al. 2018). Briefly, IPSCs (Wicell UCSD021i-3-9) were differentiated into Hematopoietic progenitor cells (HPCs) using the STEMdiff ${ }^{\mathrm{TM}}$ Hematopoietic Kit (Stem Cell Technologies \# 05310) according to manufacturer instructions. IPSCs cells were grown in mTeSR1 (STEMCELL technologies Catalog \# 85850) and passaged with $0.5 \mathrm{mM}$ EDTA in PBS $\left(-\mathrm{Ca}^{+2} / \mathrm{Mg}^{+2}\right)$. On day -1 cells were plated into mTeSR1 medium with $0.5 \mu \mathrm{M}$ Thiazovivin (STEMCELL technologies \# 72252) onto hESC-matrigel coated (Corning \# 354277) 6-well plates at different densities. On day 0 plates with a density of 10 to 20 aggregates per $\mathrm{cm}^{2}$ with a size ranging from $0.1-0.2 \mu \mathrm{m}$ were selected. A small aggregate density and size was found to be critical at this stage. mTeSR1 medium was replaced with medium A ( $2 \mathrm{~mL}$ per well of a 6-well plate). On day $250 \%$ of the medium A $(1 \mathrm{~mL} /$ well) was removed and replaced with $1 \mathrm{~mL}$ of fresh Medium A per well. On day 3, all medium was carefully removed and $2 \mathrm{~mL} /$ well medium $\mathrm{B}$ was added. Without removing media, cells were supplemented with $1 \mathrm{~mL} /$ well of medium B on days 5, 7, 9 adding the media carefully to not disturb the cells. On day 12 
non-adherent cells (HPCs) were collected carefully to not disturb adherent cells and centrifuged 300 x G 5 min. The differentiation of HPCs into IMGLs was performed using the STEMdiff ${ }^{\mathrm{TM}}$ Microglia Differentiation Kit and the STEMdiff ${ }^{\mathrm{TM}}$ Microglia Maturation Kit (STEMCELL technologies \# 100-0019 and \#100-0020) according to manufacturer instructions. Briefly, HPCs were plated at a density of 1-2 x105 cells per well in hESC-matrigel coated 6-well plates using $2 \mathrm{ml}$ of Microglia differentiation media. On day 0 until day $24,1 \mathrm{ml}$ of fresh media was added to each well, except for day 12, in which all the media except for $1 \mathrm{ml}$ was collected and spinned down $300 \mathrm{G}$ x 5min, cells were resuspended in $1 \mathrm{ml}$ of fresh media and returned to the well. On day 24 cells were collected, centrifuged 300 $\mathrm{G} \times 5 \mathrm{~min}$ and counted. $1 \mathrm{ml}$ of conditioned media per well was left and fresh Microglia Maturation Media media was added to achieve $1 \times 10^{6}$ cells in $2 \mathrm{ml}$. Cells were plated $(2 \mathrm{ml} /$ well) in fresh hESC-matrigel coated 6-well plates. $1 \mathrm{ml}$ of fresh media was added every other day until cells were used on days $28-30$. On day $28-30$ cells were semi-adherent, all media except for $1 \mathrm{ml}$ was collected, centrifuged $300 \mathrm{G} \times 5 \mathrm{~min}$ and cells were resuspended in $1 \mathrm{ml} /$ well of Microglia Maturation media with or without $2 x$ concentration of A $\beta$ or LPS/INF $\gamma$ and returned to the well. After $24 \mathrm{~h}$ treatment, cells were collected for RNA extraction.

\section{Cell labeling and $\mathrm{A} \beta$ treatment}

For live-cell imaging, 50,000 iPSC-microglia or THP1 macrophages were cultured in a vitronectin-coated eight-chambered cover glass dish (Cellvis, C8-1.5H-N). THP-1 macrophages were labeled with $2 \mu \mathrm{M}$ CellTracker Green CMFDA dye (Invitrogen, C2925) in HBSS containing $\mathrm{Ca}^{++}$and $\mathrm{Mg}^{++}$(Gibco, 14025-092) for 30 minutes at $37^{\circ} \mathrm{C}$. Medium was replaced with cell medium containing $2 \mu \mathrm{g} /$ mL $\beta$-Amyloid ${ }_{1-42}$-HiLyte Fluor 555 and cells were imaged 1 hour following treatment.

\section{Live-cell imaging}

Confocal images were acquired on an inverted Zeiss 800 laser scanning confocal microscope equipped with 405, 488, 561, and $647 \mathrm{~nm}$ diode lasers, gallium arsenide phosphide (GaAsP) detectors, and a transmitted light photomultiplier tube (PMT). Images were acquired using a $63 \mathrm{X} / 1.4 \mathrm{NA}$ objective lens at $37^{\circ} \mathrm{C}$ and $5 \% \mathrm{CO}_{2}$ (Carl Zeiss, Oberkochen, Germany).

\section{RNA-seq}

Immediately after cells were harvested, RNA was extracted using RNeasy Mini Kit (QIAGEN) following manufacturer's guidelines. RNA integrity number (RIN) was measured for all samples using Agilent tapestation 4150 system. All sequencing libraries analyzed were generated from RNA samples measuring a RIN score $\geq 8.5$. RNA-seq libraries were generated using the KAPA RNA HyperPrep with RiboErase kit using $500 \mathrm{ng}$ of isolated mRNA as input and following manufacturer's instructions. Libraries were quantified and normalized using DNA tapestation and sequenced as paired-end 75-base-pair reads in the Illumina Nextseq 500 platform.

\section{RNA-seq quantification}

RNA-seq libraries were sequenced to an average depth of approximately 55-70 million reads per sample. Low-quality reads and adapters were trimmed using Trim Galore! (v. 0.4.3), and trimmed reads were then mapped to the hg19 transcriptome (GENCODE, release 19) and quantified

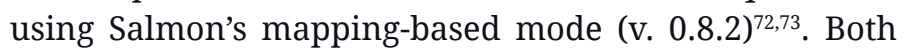
programs were run with default settings, using paired-end inputs. Gene-level quantifications were summarized from each sample using the R package tximport (v. 1.2.0) ${ }^{74}$.

\section{Principal component analysis and variance partitioning}

The untreated controls from each experiment were analyzed separately for the purpose of principal component analysis (PCA), seen in Figure 1, and characterizing drivers of variance. The read counts summarized by tximport were variance-stabilizing transformed (VST) in R using DESeq2 (v. 1.22.2) ${ }^{75}$. Genes were subset for only those with 100 or more transcripts per kilobase million (TPM) in at least one sample $(n=5,192)$. The VST counts for these genes were used to calculate variance for PC1 and PC2. Variance was characterized using the $\mathrm{R}$ package variancePartition (v. 1.22.0) using the same filtered VST counts, and the following formula: (1|Tech_Rep) + (1|Bio_Rep) $+(1 \mid$ Cell_Type $)+(1 \mid \text { Source })^{21}$. The fitExtractVarPartModel function then fits the VST counts to a linear mixed model and determines the fraction of variance attributable to each covariate in the design formula, with any unexplained variance attributed to residuals. The mean percentage of variation across all genes was reported.

\section{Differential RNA-seq analysis}

Differential analysis was conducted in R with DESeq2 (v. 1.22.2), using a design adjusting for replicate variability when calculating differences between treatment groups ( $\sim$ replicate + condition). Each experiment was analyzed separately to identify the number of differential genes caused by each treatment relative to untreated controls. Differential genes were defined as genes with an FDR-adjusted p-value below 0.01 (Wald test, log2 fold-change threshold of 1) and an absolute fold change greater than 2 when comparing treated samples to their respective controls. Fold changes were shrunken using the "apeglm" method within DESeq2 (v. 1.14.0) ${ }^{76}$.

\section{GO and KEGG Enrichment Analysis}

The "findMotifs.pl" tool in the HOMER software suite 
(v. 4.10.4) was used on genes that were upregulated in IMGLs upon LPS/INF $\gamma$-treatment to identify significantly enriched GO terms ( $<$ 0.05) and Kyoto Encyclopedia of Genes and Genomes (KEGG) pathways $(\mathrm{p}<0.05)$

\section{DAM Enrichment Analysis}

IMGL RNASeq data was subset for genes present in DAM gene signatures (DESeq2; > 100 counts per million). Fisher's exact test was used to determine the enrichment of DAM genes, using all genes expressed in IMGLs as background (DESeq2; > 10 counts per million). Wilcoxon rank sum test was used to determine whether each group was significantly different from zero.

\section{Data availability}

Raw FASTQ files are available on SRA under accession number PRJNA750801. Transcript-level quantifications from Salmon are available on GEO under series GSE181153, in addition to a single gene-level counts table with each sample represented as a column, as generated by tximport. The GEO series page also includes a table describing the samples used in DESeq2 for each column of Figure 3.

\section{Acknowledgments}

We would like to acknowledge Erika Deoudes for illustrations and graphic design. IYQ is supported by a BrightFocus Foundation postdoctoral fellowship. Acknowledgement is made to the donors of the Alzheimer's Disease Research program that supports this fellowship. AEC was supported by the UNC Postbaccalaureate Research Education Program R25GM089569. MLB is supported by the NIH/NIGMS training grant T32GM135128. BAE is supported by NSF-GRFP DGE-1650116. JVR and SC are supported by NIA, R00AG052570. RBM is supported by R01 NS108808. HW, TJC and DHP are supported by NIH grant R01AG066871.

\section{Bibliography}

1. 2020 Alzheimer's disease facts and figures. Alzheimers. Dement. (2020) doi:10.1002/alz.12068.

2. Cruts, M., Theuns, J. \& Van Broeckhoven, C. Locus-specific mutation databases for neurodegenerative brain diseases. Hum. Mutat. 33, 1340-1344 (2012).

3. Jacobs, H. I. L. et al. Structural tract alterations predict downstream tau accumulation in amyloid-positive older individuals. Nat. Neurosci. 21, 424-431 (2018).

4. Busche, M. A. \& Hyman, B. T. Synergy between amyloid- $\beta$ and tau in Alzheimer's disease. Nat. Neurosci. 23, 1183-1193 (2020).

5. Choi, S. H. et al. A three-dimensional human neural cell culture model of Alzheimer's disease. Nature 515,
274-278 (2014).

6. Schelle, J. et al. Prevention of tau increase in cerebrospinal fluid of APP transgenic mice suggests downstream effect of BACE1 inhibition. Alzheimers. Dement. 13, 701-709 (2017).

7. Bennett, R. E. et al. Enhanced Tau Aggregation in the Presence of Amyloid $\beta$. Am. J. Pathol. 187, 1601-1612 (2017).

8. Busche, M. A. et al. Tau impairs neural circuits, dominating amyloid- $\beta$ effects, in Alzheimer models in vivo. Nat. Neurosci. 22, 57-64 (2019).

9. Chételat, G. et al. Amyloid imaging in cognitively normal individuals, at-risk populations and preclinical Alzheimer's disease. Neuroimage Clin 2, 356-365 (2013).

10. Taipa, R. et al. Inflammatory pathology markers (activated microglia and reactive astrocytes) in early and late onset Alzheimer disease: a post mortem study. Neuropathol. Appl. Neurobiol. 44, 298-313 (2018).

11. Hopperton, K. E., Mohammad, D., Trépanier, M. O., Giuliano, V. \& Bazinet, R. P. Markers of microglia in post-mortem brain samples from patients with Alzheimer's disease: a systematic review. Mol. Psychiatry 23, 177-198 (2018).

12. Gomez-Nicola, D. \& Perry, V. H. Microglial dynamics and role in the healthy and diseased brain: a paradigm of functional plasticity. Neuroscientist 21, 169184 (2015).

13. Raj, D. et al. Increased White Matter Inflammation in Aging- and Alzheimer's Disease Brain. Front. Mol. Neurosci. 10, 206 (2017).

14. Yasuno, F. et al. Increased binding of peripheral benzodiazepine receptor in mild cognitive impairment-dementia converters measured by positron emission tomography with [11C]DAA1106. Psychiatry Research: Neuroimaging 203, 67-74 (2012).

15. Cagnin, A. et al. In-vivo measurement of activated microglia in dementia. Lancet 358, 461-467 (2001).

16. Mathys, H. et al. Single-cell transcriptomic analysis of Alzheimer's disease. Nature 570, 332-337 (2019).

17. Liu, C. et al. Genome-Wide Association and Mechanistic Studies Indicate That Immune Response Contributes to Alzheimer's Disease Development. Front. Genet. 9, 410 (2018).

18. Efthymiou, A. G. \& Goate, A. M. Late onset Alzheimer's disease genetics implicates microglial pathways in disease risk. Mol. Neurodegener. 12, 43 (2017).

19. Tansey, K. E., Cameron, D. \& Hill, M. J. Genetic risk for Alzheimer's disease is concentrated in specific macrophage and microglial transcriptional networks. Genome Med. 10, 14 (2018).

20. McQuade, A. et al. Development and validation of a simplified method to generate human microglia from pluripotent stem cells. Mol. Neurodegener. 13, 67 (2018). 

available under aCC-BY-NC-ND 4.0 International license.

21. Hoffman, G. E. \& Schadt, E. E. variancePartition: interpreting drivers of variation in complex gene expression studies. BMC Bioinformatics 17, 483 (2016).

22. Zhao, Y. et al. TREM2 Is a Receptor for $\beta$-Amyloid that Mediates Microglial Function. Neuron 97, 1023-1031. e7 (2018).

23. Tseng, J.-H. et al. The Deacetylase HDAC6 Mediates Endogenous Neuritic Tau Pathology. Cell Rep. 20, 2169-2183 (2017).

24. Abud, E. M. et al. iPSC-Derived Human Microglia-like Cells to Study Neurological Diseases. Neuron 94, 278293.e9 (2017).

25. Verma, M., Vats, A. \& Taneja, V. Toxic species in amyloid disorders: Oligomers or mature fibrils. Ann. Indian Acad. Neurol. 18, 138-145 (2015).

26. Vadukul, D. M. et al. Internalisation and toxicity of amyloid- $\beta$ 1-42 are influenced by its conformation and assembly state rather than size. FEBS Lett. 594, 3490-3503 (2020).

27. Stine, W. B., Jungbauer, L., Yu, C. \& LaDu, M. J. Preparing Synthetic $A \beta$ in Different Aggregation States. in Alzheimer's Disease and Frontotemporal Dementia: Methods and Protocols (ed. Roberson, E. D.) 13-32 (Humana Press, 2011).

28. Han, S.-H., Park, J.-C. \& Mook-Jung, I. Amyloid $\beta$-interacting partners in Alzheimer's disease: From accomplices to possible therapeutic targets. Prog. Neurobiol. 137, 17-38 (2016).

29. Fülöp, T., Itzhaki, R. F., Balin, B. J., Miklossy, J. \& Barron, A. E. Role of Microbes in the Development of Alzheimer's Disease: State of the Art - An International Symposium Presented at the 2017 IAGG Congress in San Francisco. Front. Genet. 9, 362 (2018).

30. Bulgart, H. R., Neczypor, E. W., Wold, L. E. \& Mackos, A. R. Microbial involvement in Alzheimer disease development and progression. Mol. Neurodegener. 15, 42 (2020).

31. Li, F., Hearn, M. \& Bennett, L. E. The role of microbial infection in the pathogenesis of Alzheimer's disease and the opportunity for protection by anti-microbial peptides. Crit. Rev. Microbiol. 47, 240-253 (2021).

32. Giridharan, V. V., Masud, F., Petronilho, F., Dal-Pizzol, F. \& Barichello, T. Infection-Induced Systemic Inflammation Is a Potential Driver of Alzheimer's Disease Progression. Front. Aging Neurosci. 11, 122 (2019).

33. Dräger, N. M. et al. A CRISPRi/a platform in iPSC-derived microglia uncovers regulators of disease states. doi:10.1101/2021.06.16.448639.

34. Sochocka, M., Zwolińska, K. \& Leszek, J. The Infectious Etiology of Alzheimer's Disease. Curr. Neuropharmacol. 15, 996-1009 (2017).

35. Naughton, S. X., Raval, U. \& Pasinetti, G. M. The Viral Hypothesis in Alzheimer's Disease: Novel Insights and Pathogen-Based Biomarkers. J Pers Med 10, (2020).
36. Batista, C. R. A., Gomes, G. F., Candelario-Jalil, E., Fiebich, B. L. \& de Oliveira, A. C. P. Lipopolysaccharide-Induced Neuroinflammation as a Bridge to Understand Neurodegeneration. Int. J. Mol. Sci. 20, (2019).

37. Lykhmus, O. et al. Molecular Mechanisms Regulating LPS-Induced Inflammation in the Brain. Front. Mol. Neurosci. 9, 19 (2016).

38. Hasselmann, J. et al. Development of a Chimeric Model to Study and Manipulate Human Microglia In Vivo. Neuron 103, 1016-1033.e10 (2019).

39. Deming, Y. et al. The MS4A gene cluster is a key modulator of soluble TREM2 and Alzheimer's disease risk. Sci. Transl. Med. 11, (2019).

40. Blumenau, S. et al. Investigating APOE, APP-A $\beta$ metabolism genes and Alzheimer's disease GWAS hits in brain small vessel ischemic disease. Sci. Rep. 10, 7103 (2020).

41. Benmamar-Badel, A., Owens, T. \& Wlodarczyk, A. Protective Microglial Subset in Development, Aging, and Disease: Lessons From Transcriptomic Studies. Front. Immunol. 11, 430 (2020).

42. Kang, S. S. et al. Microglial translational profiling reveals a convergent APOE pathway from aging, amyloid, and tau. J. Exp. Med. 215, 2235-2245 (2018).

43. Seok, J. et al. Genomic responses in mouse models poorly mimic human inflammatory diseases. Proc. Natl. Acad. Sci. U. S. A. 110, 3507-3512 (2013).

44. Heng, Y., Dubbelaar, M. L., Marie, S. K. N., Boddeke, E. W. G. M. \& Eggen, B. J. L. The effects of postmortem delay on mouse and human microglia gene expression. Glia 69, 1053-1060 (2021).

45. Gosselin, D. et al. An environment-dependent transcriptional network specifies human microglia identity. Science 356, (2017).

46. Pocock, J. M. \& Piers, T. M. Modelling microglial function with induced pluripotent stem cells: an update. Nat. Rev. Neurosci. 19, 445-452 (2018).

47. Park, J. et al. Three-dimensional brain-on-a-chip with an interstitial level of flow and its application as an in vitro model of Alzheimer's disease. Lab Chip 15, 141-150 (2015).

48. Arber, C., Lovejoy, C. \& Wray, S. Stem cell models of Alzheimer's disease: progress and challenges. Alzheimers. Res. Ther. 9, 1-17 (2017).

49. Butovsky, O. et al. Identification of a unique TGF- $\beta$-dependent molecular and functional signature in microglia. Nat. Neurosci. 17, 131-143 (2014).

50. Melief, J. et al. Characterizing primary human microglia: A comparative study with myeloid subsets and culture models. Glia 64, 1857-1868 (2016).

51. Das, A. et al. Transcriptome sequencing reveals that LPS-triggered transcriptional responses in established microglia BV2 cell lines are poorly representa- 
tive of primary microglia. J. Neuroinflammation 13 , 182 (2016).

52. Banerjee, A. et al. Validation of Induced Microglia-Like Cells (iMG Cells) for Future Studies of Brain Diseases. Front. Cell. Neurosci. 15, 629279 (2021).

53. Yates, S. L. et al. Amyloid beta and amylin fibrils induce increases in proinflammatory cytokine and chemokine production by THP-1 cells and murine microglia. J. Neurochem. 74, 1017-1025 (2000).

54. Walker, D. G., Lue, L. F. \& Beach, T. G. Gene expression profiling of amyloid beta peptide-stimulated human post-mortem brain microglia. Neurobiol. Aging 22, 957-966 (2001).

55. Vukic, V. et al. Expression of inflammatory genes induced by beta-amyloid peptides in human brain endothelial cells and in Alzheimer's brain is mediated by the JNK-AP1 signaling pathway. Neurobiol. Dis. 34, 95-106 (2009).

56. Stöhr, J. et al. Purified and synthetic Alzheimer's amyloid beta (Aß) prions. Proc. Natl. Acad. Sci. U. S. A. 109, 11025-11030 (2012).

57. Nilsson, J. et al. Synthetic standard aided quantification and structural characterization of amyloid-beta glycopeptides enriched from cerebrospinal fluid of Alzheimer's disease patients. Sci. Rep. 9, 5522 (2019).

58. Moro, M. L. et al. Pyroglutamate and Isoaspartate modified Amyloid-Beta in ageing and Alzheimer's disease. Acta Neuropathol Commun 6, 3 (2018).

59. Rasmussen, J. et al. Amyloid polymorphisms constitute distinct clouds of conformational variants in different etiological subtypes of Alzheimer's disease. Proc. Natl. Acad. Sci. U. S. A. 114, 13018-13023 (2017).

60. Paravastu, A. K., Qahwash, I., Leapman, R. D., Meredith, S. C. \& Tycko, R. Seeded growth of beta-amyloid fibrils from Alzheimer's brain-derived fibrils produces a distinct fibril structure. Proc. Natl. Acad. Sci. U. S. A. 106, 7443-7448 (2009).

61. Neddens, J. et al. Correlation of pyroglutamate amyloid $\beta$ and ptau Ser202/Thr205 levels in Alzheimer's disease and related murine models. PLoS One 15, e0235543 (2020).

62. Perez-Garmendia, R. \& Gevorkian, G. Pyroglutamate-Modified Amyloid Beta Peptides: Emerging Targets for Alzheimer's Disease Immunotherapy. Curr. Neuropharmacol. 11, 491-498 (2013).

63. Esparza, T. J. et al. Soluble Amyloid-beta Aggregates from Human Alzheimer's Disease Brains. Sci. Rep. 6, 38187 (2016).

64. Liu, J., Qian, C. \& Cao, X. Post-Translational Modification Control of Innate Immunity. Immunity 45, 15-30 (2016).

65. Anderson, P. Post-transcriptional control of cytokine production. Nat. Immunol. 9, 353-359 (2008).

66. Mino, T. \& Takeuchi, O. Post-transcriptional regula- tion of cytokine mRNA controls the initiation and resolution of inflammation. Biotechnol. Genet. Eng. Rev. 29, 49-60 (2013).

67. Sondag, C. M., Dhawan, G. \& Combs, C. K. Beta amyloid oligomers and fibrils stimulate differential activation of primary microglia. J. Neuroinflammation 6 , 1 (2009).

68. McLarnon, J. G. Correlated inflammatory responses and neurodegeneration in peptide-injected animal models of Alzheimer's disease. Biomed Res. Int. 2014, 923670 (2014).

69. Leung, E. et al. Microglia activation mediates fibrillar amyloid- $\beta$ toxicity in the aged primate cortex. Neurobiol. Aging 32, 387-397 (2011).

70. Jana, M., Palencia, C. A. \& Pahan, K. Fibrillar amyloid-beta peptides activate microglia via TLR2: implications for Alzheimer's disease. J. Immunol. 181, 7254-7262 (2008).

71. Daly, T., Houot, M., Barberousse, A., Petit, A. \& Epelbaum, S. A proposal to make biomedical research into Alzheimer's disease more democratic following an international survey with researchers. J. Alzheimers Dis. Rep. 1-9 (2021).

72. Babraham Bioinformatics - Trim Galore! http://www. bioinformatics.babraham.ac.uk/projects/trim_galore/.

73. Patro, R., Duggal, G., Love, M. I., Irizarry, R. A. \& Kingsford, C. Salmon provides fast and bias-aware quantification of transcript expression. Nat. Methods 14, 417-419 (2017).

74. Soneson, C., Love, M. I. \& Robinson, M. D. Differential analyses for RNA-seq: transcript-level estimates improve gene-level inferences. F1000Res. 4, 1521 (2015).

75. Love, M. I., Huber, W. \& Anders, S. Moderated estimation of fold change and dispersion for RNA-seq data with DESeq2. Cold Spring Harbor Laboratory 002832 (2014) doi:10.1101/002832.

76. Zhu, A., Ibrahim, J. G. \& Love, M. I. Heavy-tailed prior distributions for sequence count data: removing the noise and preserving large differences. Bioinformatics 35, 2084-2092 (2019). 
bioRxiv preprint doi: https://doi.org/10.1101/2021.09.14.460110; this version posted September 15, 2021. The copyright holder for this preprint (which was not certified by peer review) is the author/funder, who has granted bioRxiv a license to display the preprint in perpetuity. It is made available under aCC-BY-NC-ND 4.0 International license.

\section{Supplemental Information}

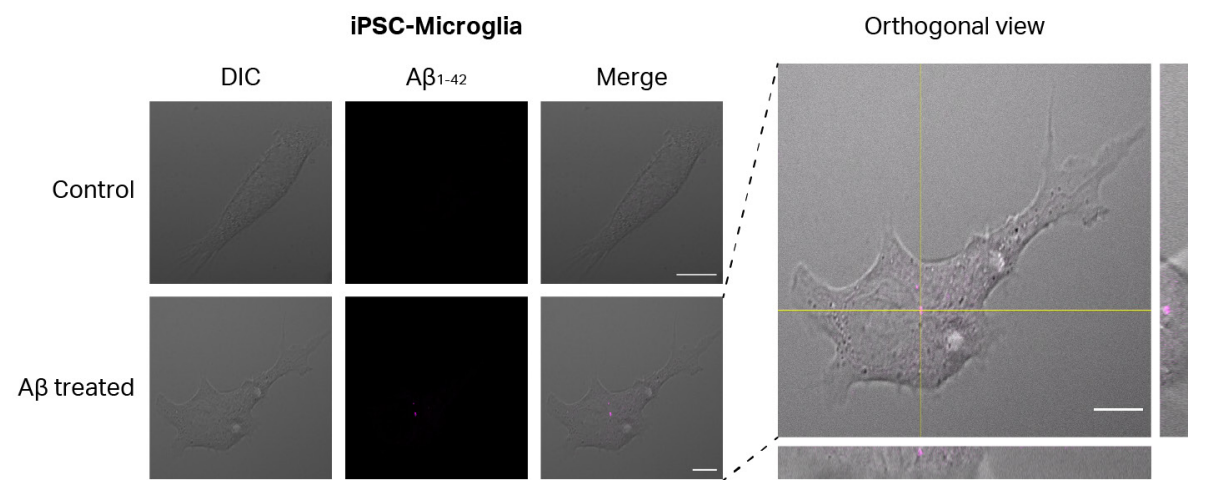

Supplementary Fig. 1 | A $\beta$ is incorporated into IMGLs. IMGLs incubated with $2 \mu \mathrm{g} / \mathrm{mL}$ of oligomeric $\beta$-Amyloid $_{1-42}$-HiLyte Fluor 555 for $1 \mathrm{hr}$. Confocal images were acquired on an inverted Zeiss 800 laser scanning confocal microscope. Scale bar, $10 \mu \mathrm{m}$. 